\title{
SPATIAL AND TEMPORAL VARIATION IN DENSITIES OF CHIRONOMID LARVAE (DIPTERA) IN TWO LAGOONS AND TWO TRIBUTARIES OF THE UPPER PARANÁ RIVER FLOODPLAIN, BRAZIL
}

\author{
HIGUTI, J. and TAKEDA, A. M. \\ Universidade Estadual de Maringá, Av. Colombo, 5790, Nupélia, Bloco H-90, \\ CEP 87020-900, Maringá, PR, Brazil \\ Correspondence to: Janet Higuti, Universidade Estadual de Maringá, Av. Colombo, 5790, Nupélia, Bloco H-90, \\ CEP 87020-900, Maringá, PR, Brazil, e-mail: higuti@ nupelia.uem.br \\ Received January 18, 2002 - Accepted April 20, 2002 - Distributed November 30, 2002
}

(With 5 figures)

\begin{abstract}
Chironomid larvae were collected and abiotic variables measured at monthly intervals at 21 sampling stations in two lagoons (Guaraná and Patos) and two tributaries (Baía and Ivinheima) of the Upper Paraná River floodplain. The genera Procladius, Chironomus, Goeldichironomus, and Polypedilum were dominant in the lagoons and in the Baía River, while Cryptochironomus and Lopescladius were more dominant in the Ivinheima River. The similarity in the generic composition of the chironomids of the lagoons and the Baía River is probably due to the fact that this river presents hydrodynamic characteristics similar to those of the lagoons. The results obtained suggest that the hydrodynamics of these environments are the main factor determining spatial variation in the chironomid fauna. This is intuitively clear, as this factor itself determines sediment type, quantity of organic matter and presence or absence of aquatic macrophytes. However, on a seasonal scale, the flood pulse seems to be the main controlling factor of the temporal variation in densities and dominance of chironomid larvae. Given that this factor has a large influence on the temporal dynamics of several limnological variables, this is again a logical correlation. Our results suggest a strong relationship between the variations in the chironomid community and fluctuations in limnological characteristics.
\end{abstract}

Key words: Chironomidae, zoobenthos, floodplain, spatial and temporal variation.

\section{RESUMO}

\section{Variação espacial e temporal da densidade de larvas de quironomídeos (Diptera) em duas} lagoas e dois tributários da planície de inundação do alto rio Paraná, Brasil

Foram coletadas larvas de quironomídeos e medidas as variáveis abióticas, mensalmente, em 21 pontos distribuídos em duas lagoas (Guaraná e Patos) e dois tributários (Baía e Ivinheima) da planície de inundação do alto rio Paraná. Os gêneros Procladius, Chironomus, Goeldichironomus e Polypedilum foram dominantes nas lagoas e no rio Baía, enquanto Cryptochironomus e Lopescladius dominaram no rio Ivinheima. Essa semelhança na composição genérica de quironomídeos nas lagoas e no rio Baía provavelmente se deve ao fato de esse rio apresentar características hidrodinâmicas mais similares às das lagoas. Os resultados obtidos sugerem que a hidrodinâmica desses ambientes é o principal fator determinante na variação espacial da fauna de quironomídeos. Obviamente, esse fator determina o tipo de sedimento, teor de matéria orgânica e presença ou ausência de macrófitas aquáticas. No entanto, sazonalmente, o pulso de inundação parece ser o principal fator controlador da variação temporal da densidade e da dominância das larvas de quironomídeos. Tendo em vista que esse fator tem grande influência na dinâmica temporal das variáveis limnológicas, isso é novamente uma correlação lógica. Os resultados sugerem forte relação entre as variações na comunidade de quironomídeos e as flutuações nas características limnológicas.

Palavras-chave: Chironomidae, zoobentos, planície, variação espacial e temporal. 


\section{INTRODUCTION}

The large rivers in South America are characterised by extensive floodplains, which can significantly influence the dynamics and the productivity of the river beds themselves, as well as their adjacent environments (Bonetto, 1993). Floodplains are mostly very productive and include riparian vegetation and a large diversity of aquatic microhabitats (Amoros, 1991). These areas have high biological activity, mainly in the shallow water ecotones (Junk et al., 1989). It has been shown that the composition and structure of ecotone communities are more sensitive to hydrological fluctuations than to changes in the availability of nutrients (Dokulil \& Schiel, 2000). Fluctuations in the benthic communities of different biotopes of the Upper Paraná River floodplain are mainly characterised by changes in frequency and abundance of larval chironomid populations (Takeda et al., 1990, 1991a, 1991b; Higuti et al., 1993). Their rapid turnover of generations and their fast growth rate (Menzie, 1981), furthermore gives an important role to these organisms in the energy flux of the ecosystem (Coffman \& Ferrington, 1988). The aim of the present study was to investigate the spatial and temporal variations in the density and dominance of chironomid larvae and the relationship of these variations to limnological factors in two lagoons and two rivers of the Upper Paraná River floodplain.

\section{MATERIAL AND METHODS}

\section{Description of study sites}

The Baía River (2243'21'S and 53¹8'42”W)

comprises numerous lagoons along its course, including the Guaraná lagoon (22\%43'26'S and $\left.53^{\circ} 18^{\prime} 03^{\prime \prime} \mathrm{W}\right)$. This lagoon is situated on the right bank of the lower end of the river to which it is connected by a short and narrow channel. The vegetation around the lagoon is composed of Panicum prionitis and Polygonum spp. and in the littoral zone Eichhornia crassipes, Pistia stratiotes, Salvinia sp., and mainly Eichhornia azurea.

The Ivinheima River (22\%49'01'S and $53^{\circ} 33^{\prime} 42^{\prime \prime} \mathrm{W}$ ) is the main tributary on the right bank of the Paraná River in the study area. The Patos lagoon (22.49'19'S and 53'31'33'W) is located on the left bank of the Ivinheima River, to which it is permanently connected by a long channel. In this lagoon, the marginal vegetation is composed mainly of Polygonum spp., while the littoral is colonised by E. azurea.

The sampling sites were divided into, littoral region: G1, G5, G6, G7 (Guaraná lagoon) and P1, P5, P6, P7 (Patos lagoon); profundal zone: G2, G3, G4 (Guaraná lagoon) and P2, P3, P4 (Patos lagoon); margins: B1, B5 (Baía River) and I1, I2 (Ivinheima River); and the central region: B2, B3, B4 (Baía River) (Fig. 1).

\section{Sampling and proceeding}

Zoobenthos was sampled and physical and chemical variables of the water were measured monthly between March 1993 and February 1994.

At each sampling site, three benthos samples were collected and fixed with $10 \%$ buffered formalin and one sample was collected for sedimentological analyses. All were taken with a modified Petersen grab $\left(0.0345 \mathrm{~m}^{2}\right)$. A $200 \mu \mathrm{m}$ sieve was used to separate the organisms from sediment. Chironomids larvae were later sorted and identified down to genus level.

Water transparency was measured with a Secchi disc and water temperature were measured with a thermistor. Bottom water samples were collected with a Van Dorn bottle. $\mathrm{pH}$ and electrical conductivity were measured using portable Digimed meters. Dissolved oxygen was determined using the Winkler modified as described by Golterman et al. (1978).

Sediment granulometry was determined according to the method of Suguio (1973) using the scale of Wentworth (1922); organic matter in the sediment was obtained from $10 \mathrm{~g}$ sediment by incineration at $560^{\circ} \mathrm{C}$ during four hours.

The daily water level data from the Paraná and Ivinheima Rivers were provided by the National Department of Water and Eletrical Energy (DNAEE). For both rivers we considered the mean hydrological level of the 6 days preceding sampling according to Thomaz et al. (1997). 


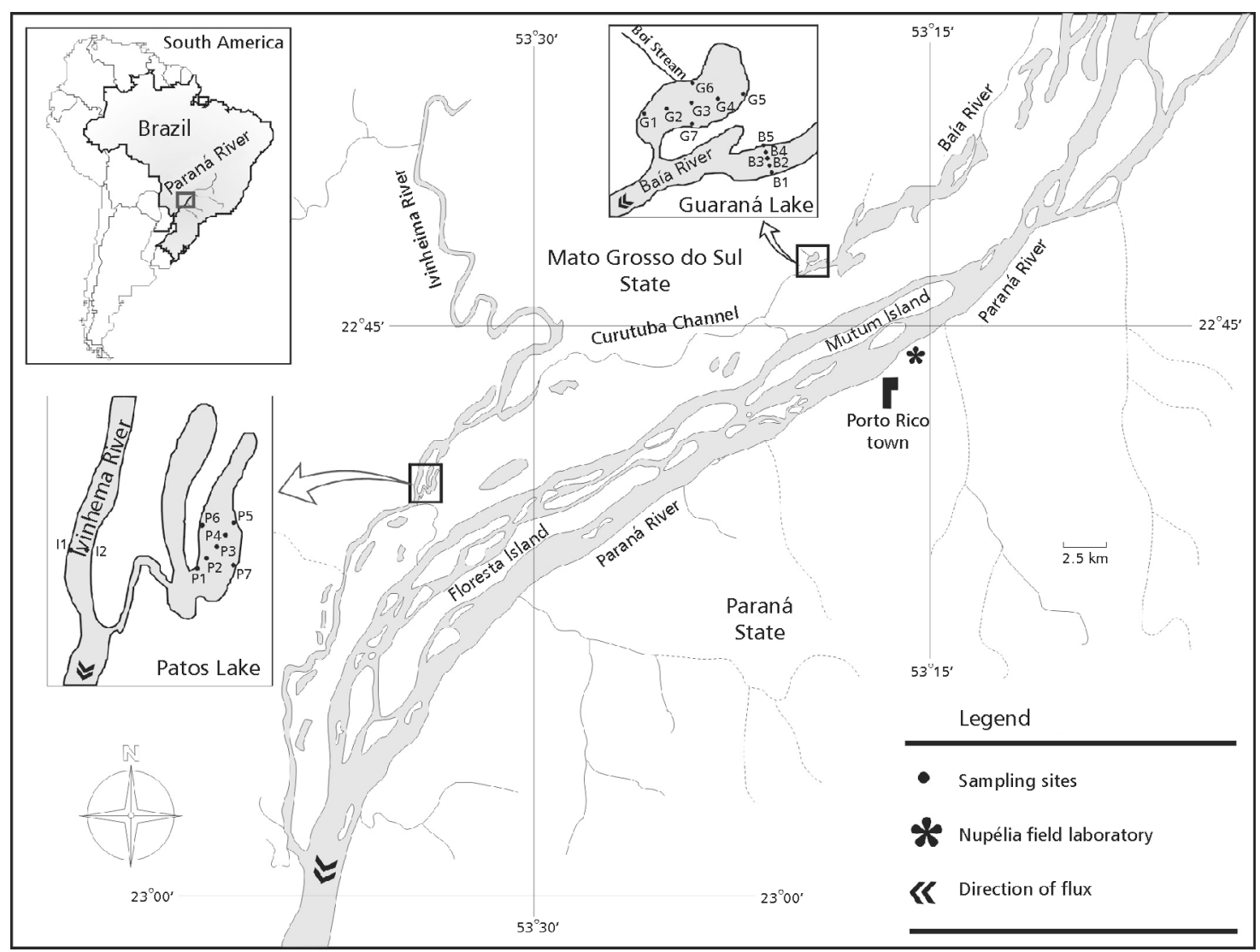

Fig. 1 - Study area and sampling sites.

\section{Data analyses}

Kownacki (1971) dominance indices were calculated for all sites, in two hydrological periods (high and low water) and using the mean density of chironomids larvae. Principal Components Analysis (PCA) and Detrended Correspondence Analysis (DCA) were applied to reduce of the dimensionality of the abiotic data set (depth, water temperature, water transparency, $\mathrm{pH}$, electrical conductivity, dissolved oxygen, level water, granulometry and organic matter of the sediment) and biotic data (density and diversity of chironomid larvae), respectively. The abiotic data were standardised $(\mathrm{x}=\mathrm{x}-\bar{x} / \mathrm{sd})$, except $\mathrm{pH}$, and the biotic data were $\log$ transformed $[\log (x+1)]$, to minimise the effect of discrepant values. Twenty chironomid genera were considered in the DCA. Only the occurrence of genera with more than 10 individuals were taken into consideration; this criterion was used to reduce the effect of rare genera.

The Procustean Randomisation Test - Protest (Jackson, 1995) was carried out using PCA and DCA scores to evaluate the relationship between spatial and temporal pattern of environment variables and chironomids assemblages. In Procrustes analysis, a pair of data matrices is compared by using a rotational-fit algorithm that minimises the sum-ofthe- squared residuals between the two matrices, i.e. the $\mathrm{m}^{2}$ statistic. Residuals between the original values and the best-fit solution are calculated for each location or observation to identify outlying and deviant points. The resultant $\mathrm{m}^{2}-$ value is a goodness-of-fit statistic that describes the degree of concordance between two matrices. Jackson (1995) proposed the use of a randomization test to evaluate the significance of an observed $\mathrm{m}^{2}$ statistic. 


\section{RESULTS}

The Baía River and Guaraná lagoon are significantly influenced by the hydrometric level of the Paraná River. The Patos lagoon is influenced by the hydrometric level of the Ivinheima River. Periods with high water in the Paraná and Ivinheima Rivers were arbitrarily delimited when the depth was larger than 3.5 and 2.0 meters, respectively (Fig. 2).

The depth of the sampling sites varied between 0.6 (river margins) and $6.2 \mathrm{~m}$ (Patos lagoon profundal zone and Baía River central area). The greater percentages of organic matter were found in the Baía River margin (16\%), Guaraná lagoon littoral region and Patos lagoon profundal zone $(15 \%)$. The dissolved oxygen mean values were higher in the marginal zones of the Baía (8.5 mg.L $\mathrm{L}^{-1}$ ) and Ivinheima (7.6 mg.L $\mathrm{L}^{-1}$ ) Rivers, while in the profundal zones of the lagoons, less oxygen was found during the high water period (Guaraná lagoon: $-0.8 \mathrm{mg} . \mathrm{L}^{-1}$, and Patos lagoon: $1.2 \mathrm{mg} . \mathrm{L}^{-1}$ ). Electrical conductivity varied between 17 and 52 $\mu \mathrm{S} . \mathrm{cm}^{-1}$,with higher values occurring during the high water period. $\mathrm{pH}$ varied from slightly acid (5.3) to slightly alkaline (8.4) (Table 1).

The chironomid larvae (3.023 individuals) collected were represented by thirty three genera and three subfamilies. In the lagoons, greater mean densities were observed during low water period, and this mainly in the littoral region, especially in
Patos lagoon. In the Guaraná lagoon littoral zone, Procladius (dominance index $=10.7$ ) was dominant during high water period and Polypedium (dominance index $=17.9$ ) during low water period. There was no dominance effect in the profundal zone.

The greater mean densities of chironomids were observed in the marginal areas of the rivers in both hydrological periods, but mainly in Patos lagoon during low water. Chironomus (dominance index $=25.1$ ) was most dominant in Patos lagoon during high water and Polypedilum (dominance index $=36.8$ ) during low water period. Goeldichironomus was dominant in the marginal area and Procladius in the central region of the Baía River, in both hydrological periods. In the Ivinheima River, Cryptochironomus (dominance index $=11.7$ ) was dominant during low water period and Lopescladius (dominance index $=15.1$ ) during high water period (Table 2).

The first two axes of Principal Components Analysis (PCA) explain $38.4 \%$ of the total variance of the physical and chemical variables. The variables that presented strong correlation $(\geq 0.5)$ in the first axis were very coarse sand $(r=0.74)$, coarse sand $(\mathrm{r}=0.73)$, medium sand $(\mathrm{r}=0.66)$, granules $(\mathrm{r}=$ $0.59)$, mud $(r=-0.72)$ and organic matter $(r=-0.64)$. On the second PCA axis, a positive correlation was found with water level $(\mathrm{r}=0.60)$, water temperature $(\mathrm{r}=0.59)$, electrical conductivity $(\mathrm{r}=0.56)$ and depth $(\mathrm{r}=0.54)$, and a negative correlation with dissolved oxygen $(r=-0.86)$.

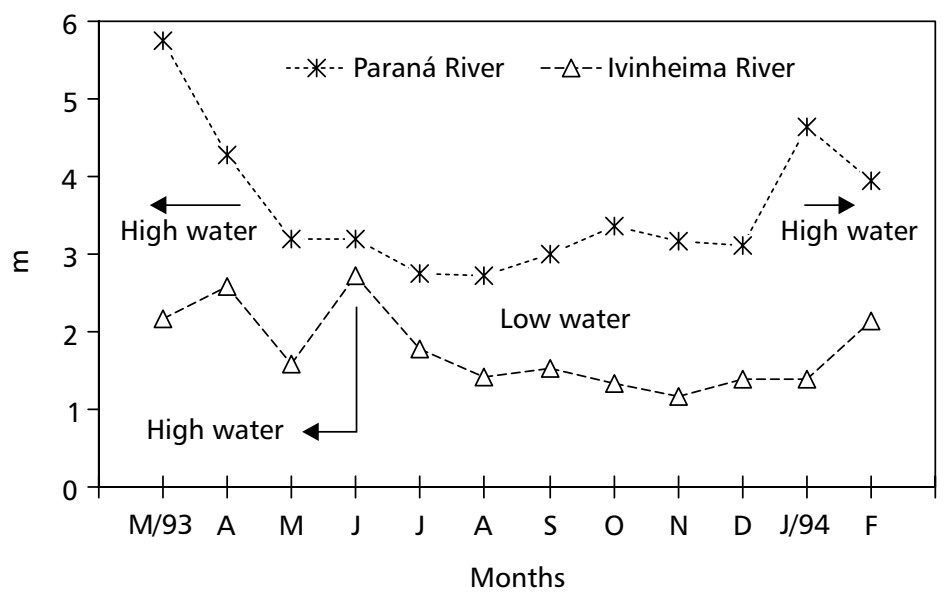

Fig. 2 - Water level (mean values of 6 days preceding sampling) of the Paraná and Ivinheima Rivers. 
TABLE 1

Mean values, standard deviation (in parenthesis), variation amplitude and number of observations (n) of the physical and chemical variables.

\begin{tabular}{|c|c|c|c|c|c|c|c|}
\hline $\begin{array}{c}\text { Sampling } \\
\text { stations }\end{array}$ & $\begin{array}{c}\text { Depth } \\
\text { (m) }\end{array}$ & $\begin{array}{c}\text { Water } \\
\text { temperature } \\
\left({ }^{\circ} \mathrm{C}\right) \\
\end{array}$ & $\begin{array}{c}\text { Water } \\
\text { transparency } \\
(\mathbf{m})\end{array}$ & pH & $\begin{array}{c}\text { Electrical } \\
\text { conductivity } \\
\left(\mu \mathrm{S} . \mathrm{cm}^{-1}\right)\end{array}$ & $\begin{array}{c}\text { Dissolved } \\
\text { oxygen } \\
\left(\mathrm{mg.L}^{-1}\right)\end{array}$ & $\begin{array}{c}\text { Organic } \\
\text { matter } \\
(\%)\end{array}$ \\
\hline \multicolumn{8}{|c|}{ Guaraná Lagoon } \\
\hline Littoral region & $2.4(0.6)$ & $25.9(2.4)$ & $1.3(0.7)$ & $6.1(0.4)$ & $38.5(7.6)$ & $1.2(0.8)$ & $15.0(13.6)$ \\
\hline \multirow[t]{3}{*}{ High water } & $1.8-3.4$ & $21.9-28.7$ & $0.3-2.9$ & $5.4-6.6$ & $25-47$ & $0.27-3.26$ & $1.14-36.3$ \\
\hline & $\mathrm{n}=16$ & $\mathrm{n}=16$ & $\mathrm{n}=16$ & $\mathrm{n}=16$ & $\mathrm{n}=16$ & $\mathrm{n}=16$ & $\mathrm{n}=16$ \\
\hline & $1.1(0.3)$ & $21.5(2.7)$ & $0.8(0.2)$ & $6.0(0.3)$ & $24.6(4.0)$ & $5.6(2.1)$ & $11.1(11.9)$ \\
\hline \multirow[t]{2}{*}{ Low water } & $0.8-2.0$ & $16.8-26.5$ & $0.5-1.2$ & $5.5-6.6$ & $21-33$ & $2.38-9.37$ & $1.3-36.6$ \\
\hline & $\mathrm{n}=32$ & $\mathrm{n}=32$ & $\mathrm{n}=32$ & $\mathrm{n}=32$ & $\mathrm{n}=32$ & $\mathrm{n}=32$ & $\mathrm{n}=32$ \\
\hline $\begin{array}{l}\text { Profundal } \\
\text { region }\end{array}$ & $3.6(0.6)$ & $24.9(2.3)$ & $1.3(0.7)$ & $5.9(0.3)$ & $40(6.4)$ & $0.8(0.6)$ & $7.7(6.6)$ \\
\hline \multirow[t]{3}{*}{ High water } & $2.8-4.6$ & $21.3-27.1$ & $0.35-2.05$ & $5.3-6.4$ & $30-47$ & $0.30-2.07$ & $1.6-26$ \\
\hline & $\mathrm{n}=12$ & $\mathrm{n}=12$ & $\mathrm{n}=12$ & $\mathrm{n}=12$ & $\mathrm{n}=12$ & $\mathrm{n}=12$ & $\mathrm{n}=12$ \\
\hline & $2.1(0.3)$ & $21(2.5)$ & $0.8(0.2)$ & $6.0(0.3)$ & $24.6(3.9)$ & $5.5(2.1)$ & $6.0(3.8)$ \\
\hline \multirow[t]{2}{*}{ Low water } & $1.6-2.8$ & $16.9-26.1$ & $0.65-1.20$ & $5.5-6.7$ & $21-33$ & $2.05-9.03$ & $1.8-15.5$ \\
\hline & $\mathrm{n}=24$ & $\mathrm{n}=24$ & $\mathrm{n}=24$ & $\mathrm{n}=24$ & $\mathrm{n}=24$ & $\mathrm{n}=24$ & $\mathrm{n}=24$ \\
\hline \multicolumn{8}{|l|}{ Baía River } \\
\hline $\begin{array}{l}\text { Marginal } \\
\text { region }\end{array}$ & $1.9(0.6)$ & $27.5(2.9)$ & $1.2(0.5)$ & $6.5(0.5)$ & $35.5(8.4)$ & $5.1(2.5)$ & $16.0(5.8)$ \\
\hline \multirow[t]{3}{*}{ High water } & $1.2-3.0$ & $23.2-30.7$ & $0.50-1.95$ & $5.6-6.9$ & $23-46$ & $2.12-7.60$ & $5.5-22.6$ \\
\hline & $\mathrm{N}=8$ & $\mathrm{n}=8$ & $\mathrm{n}=8$ & $\mathrm{n}=8$ & $\mathrm{n}=8$ & $\mathrm{n}=8$ & $\mathrm{n}=8$ \\
\hline & $1.2(0.4)$ & $22.6(2.9)$ & $0.9(0.1)$ & $6.8(0.6)$ & $22.1(3.1)$ & $8.5(1.1)$ & $14.4(7.3)$ \\
\hline \multirow[t]{2}{*}{ Low water } & $0.6-2.0$ & $17.9-28.3$ & $0.65-1.05$ & $6.0-8.4$ & $17-27$ & $6.83-10.60$ & $4.8-25$ \\
\hline & $\mathrm{n}=16$ & $\mathrm{n}=16$ & $\mathrm{n}=16$ & $\mathrm{n}=16$ & $\mathrm{n}=16$ & $\mathrm{n}=16$ & $\mathrm{n}=16$ \\
\hline Central region & $4.5(1.1)$ & $27.1(2.7)$ & $1.3(0.4)$ & $6.4(0.4)$ & $35.1(8.3)$ & $4.3(2.6)$ & $7.2(2.1)$ \\
\hline \multirow[t]{3}{*}{ High water } & $3.2-6.2$ & $22.8-30.5$ & $0.7-2.0$ & $5.7-7.0$ & $23-46$ & $0.65-7.05$ & $4.6-10.9$ \\
\hline & $\mathrm{n}=11$ & $\mathrm{n}=12$ & $\mathrm{n}=10$ & $\mathrm{n}=12$ & $\mathrm{n}=12$ & $\mathrm{n}=12$ & $\mathrm{n}=12$ \\
\hline & $3.1(1.3)$ & $21.7(2.7)$ & $1.0(0.2)$ & $6.7(0.5)$ & $22.0(3.2)$ & $8.1(1.1)$ & $6.7(4.9)$ \\
\hline \multirow[t]{2}{*}{ Low water } & $1.8-5.4$ & $17.2-27.0$ & $0.65-1.40$ & $5.9-7.7$ & $17-27$ & $6.49-9.65$ & $0.6-25.3$ \\
\hline & $\mathrm{n}=24$ & $\mathrm{n}=24$ & $\mathrm{n}=24$ & $\mathrm{n}=24$ & $\mathrm{n}=24$ & $\mathrm{n}=24$ & $\mathrm{n}=24$ \\
\hline \multicolumn{8}{|l|}{ Patos Lagoon } \\
\hline Littoral region & $2.5(1.0)$ & $25.6(4.4)$ & $1.5(1.0)$ & $6.2(0.4)$ & $39.2(8.7)$ & $1.5(0.8)$ & $11.4(5.6)$ \\
\hline \multirow[t]{3}{*}{ High water } & $1.2-4.6$ & $17.9-29.9$ & $0.55-2.95$ & $5.7-7.0$ & $25-49$ & $0.63-3.34$ & $2.4-23.4$ \\
\hline & $\mathrm{n}=16$ & $\mathrm{n}=16$ & $\mathrm{n}=16$ & $\mathrm{n}=16$ & $\mathrm{n}=16$ & $\mathrm{n}=16$ & $\mathrm{n}=16$ \\
\hline & $1.6(0.6)$ & $22.4(2.3)$ & $0.9(0.4)$ & $6.6(0.3)$ & $31.5(6.8)$ & $7.1(1.7)$ & $11.5(4.8)$ \\
\hline \multirow[t]{2}{*}{ Low water } & $0.8-3.0$ & $19.2-26.6$ & $0.30-1.65$ & $6.1-7.5$ & $23-41$ & $2.2-9.4$ & $1.95-18.70$ \\
\hline & $\mathrm{n}=32$ & $\mathrm{n}=32$ & $\mathrm{n}=32$ & $\mathrm{n}=32$ & $\mathrm{n}=32$ & $\mathrm{n}=32$ & $\mathrm{n}=32$ \\
\hline $\begin{array}{l}\text { Profundal } \\
\text { region }\end{array}$ & $4.9(0.8)$ & $24.7(5.1)$ & $1.5(1.0)$ & $6.2(0.4)$ & $42.5(9.9)$ & $1.2(0.5)$ & $15.0(6.0)$ \\
\hline \multirow[t]{3}{*}{ High water } & $3.7-6.2$ & $16.1-29.7$ & $0.55-2.90$ & $5.6-6.8$ & $26-52$ & $0.36-2.04$ & $2.9-22.5$ \\
\hline & $\mathrm{n}=12$ & $\mathrm{n}=12$ & $\mathrm{n}=12$ & $\mathrm{n}=12$ & $\mathrm{n}=12$ & $\mathrm{n}=12$ & $\mathrm{n}=12$ \\
\hline & $3.9(0.7)$ & $21.5(2.3)$ & $1.0(0.5)$ & $6.5(0.3)$ & $31.3(6.9)$ & $6.6(1.7)$ & $15.0(5.7)$ \\
\hline \multirow[t]{2}{*}{ Low water } & $2.8-5.4$ & $18.5-26.3$ & $0.45-2.10$ & $5.9-7.1$ & $23-42$ & $2.95-9.24$ & $1.7-25.9$ \\
\hline & $\mathrm{n}=24$ & $\mathrm{n}=24$ & $\mathrm{n}=24$ & $\mathrm{n}=24$ & $\mathrm{n}=24$ & $\mathrm{n}=24$ & $\mathrm{n}=24$ \\
\hline \multicolumn{8}{|c|}{ Ivinheima River } \\
\hline $\begin{array}{l}\text { Marginal } \\
\text { region }\end{array}$ & $2.7(1.6)$ & $24.7(5.0)$ & $0.9(0.7)$ & $6.7(0.3)$ & $41.3(4.0)$ & $6.4(1.2)$ & $5.2(4.8)$ \\
\hline \multirow[t]{3}{*}{ High water } & $0.8-5.8$ & $17.1-30.0$ & $0.4-2.5$ & $6.3-7.1$ & $35-44$ & $4.54-8.12$ & $0.8-15.5$ \\
\hline & $\mathrm{n}=7$ & $\mathrm{n}=7$ & $\mathrm{n}=7$ & $\mathrm{n}=7$ & $\mathrm{n}=7$ & $\mathrm{n}=7$ & $\mathrm{n}=7$ \\
\hline & $1.6(0.9)$ & $22.8(2.3)$ & $0.7(0.2)$ & $6.9(0.2)$ & $42.6(2.7)$ & $7.6(0.9)$ & $2.5(1.4)$ \\
\hline \multirow[t]{2}{*}{ Low water } & $0.6-3.8$ & $20.2-27.5$ & $0.45-1.20$ & $6.6-7.1$ & $38-47$ & $6.15-8.64$ & $0.8-6.1$ \\
\hline & $\mathrm{n}=16$ & $\mathrm{n}=16$ & $\mathrm{n}=16$ & $\mathrm{n}=16$ & $\mathrm{n}=16$ & $\mathrm{n}=16$ & $\mathrm{n}=16$ \\
\hline
\end{tabular}


TABLE 2

Values of chironomid larvae mean density $\left(\right.$ ind. $\left.\mathrm{m}^{-2}\right)$, standard deviation (in parenthesis) and dominance. $(\mathrm{G}=$ Guaraná lagoon; B = Baía River; P = Patos lagoon; $\mathbf{I}=$ Ivinheima River; $\mathbf{I}=$ littoral region; $\mathbf{p}=$ profundal region; $\mathbf{m}=$ marginal region; $c=$ central region; $a=$ high water period; $b=$ low water period $)$. Dominant $(10-100)$ values in bold letters; subdominant (1-9,99), and not dominant (0-0,99); according to Kownacki (1971).

\begin{tabular}{|c|c|c|c|c|c|c|c|c|}
\hline & Gla & Glb & Gpa & Gpb & Bma & Bmb & Bca & Bcb \\
\hline & $\chi(s) \mathrm{d}$ & $\chi(\mathrm{s}) \mathrm{d}$ & $\chi(\mathrm{s}) \mathrm{d}$ & $\chi(\mathrm{s}) \mathrm{d}$ & $\chi(\mathrm{s}) \mathrm{d}$ & $\chi(\mathrm{s}) \mathrm{d}$ & $\chi(\mathrm{s}) \mathrm{d}$ & $\chi(\mathrm{s}) \mathrm{d}$ \\
\hline \multicolumn{9}{|l|}{ Tanypodinae } \\
\hline Ablabesmyia & & & & & $8.4(15.8) 1.5$ & $0.6(2.4) 0.1$ & $0.8(2.7) 0.1$ & $0.4(1.9) 0.1$ \\
\hline Clinotanypus & & & & & & & & $0.8(2.7) 0.1$ \\
\hline Coelotanypus & $1.2(4.8) 2.4$ & $0.6(3.4) 0.1$ & $1.6(3.7) 8.4$ & & $3.6(5) 1$ & $7.2(15.5) 0.7$ & $8(10.7) 7.6$ & $5.6(18) 1.4$ \\
\hline \multicolumn{9}{|l|}{ Djalmabatista } \\
\hline Labrundinia & & & & & & & $0.8(2.7) 0.1$ & $0.4(1.9) 0.1$ \\
\hline Procladius & $1.8(3.8) \mathbf{1 0 . 7}$ & $4.5(16) 1.9$ & $0.8(2.7) 2$ & $1.6(4.6) 3.8$ & $30.1(44.8) \mathbf{1 4 . 2}$ & $4.2(8.6) 0.3$ & $22.5(34.3) 29.5$ & $42.6(58.5) 42.7$ \\
\hline \multicolumn{9}{|l|}{ Chironominae } \\
\hline Aedokritus & & $0.6(2.3) 0.1$ & & & $6(11.4) 1.1$ & $13.2(27) 1.1$ & $2.4(8.3) 0.4$ & $6.8(27.6) 1$ \\
\hline Axarus & & & & & $6(11.4) 1.1$ & $18.1(72.4) 0.3$ & & \\
\hline Beardius & & & & & & $0.6(2.4) 0.1$ & & \\
\hline Chironomus & & $2.4(6) 1.2$ & & $0.8(2.7) 1.2$ & & $3.0(4.6) 0.3$ & $5.6(11.9) 3.1$ & $2.0(4) 0.5$ \\
\hline \multicolumn{9}{|l|}{ Cladopelma } \\
\hline Cryptochironomus & & & & $0.4(1.9) 0.3$ & $1.2(3.4) 0.1$ & $2.4(7.4) 0.1$ & $0.8(2.7) 0.1$ & $1.6(6.1) 0.1$ \\
\hline Dicrotendipes & & & & & & $0.6(2.4) 0.1$ & & \\
\hline \multicolumn{9}{|l|}{ Fissimentum sp2 } \\
\hline \multicolumn{9}{|l|}{ F. desiccatum } \\
\hline Goeldichironomus & & $3.6(13.1) 0.9$ & & $1.2(4.3) 1.8$ & $62.8(117.3) 17.7$ & $103.2(236.8) 24.4$ & $0.8(2.7) 0.1$ & $1.6(6.1) 0.1$ \\
\hline Harnischia & & $0.3(1.7) 0.1$ & & & $1.2(3.4) 0.1$ & & & $0.4(1.9) 0.1$ \\
\hline Nimbocera & & & & & & $4.8(12.7) 0.3$ & & $3.6(6.8) 1$ \\
\hline Parachironomиs & & $0.3(1.7) 0.1$ & & $0.4(1.9) 0.3$ & $7.2(16.9) 1.3$ & $0.6(2.4) 0.1$ & & \\
\hline Polypedilum & & $22.9(67) 17.9$ & $0.8(2.7) 2$ & $0.4(1.9) 0.3$ & $2.4(4.4) 0.4$ & $93(257.4) 18$ & $1.6(5.5) 0.2$ & $9.6(24.8) 2.4$ \\
\hline \multicolumn{9}{|l|}{ Rheotanytarsus } \\
\hline Saetheria (?) & & & & & $1.2(3.4) 0.1$ & & $0.8(2.7) 0.1$ & $5.2(16.6) 0.7$ \\
\hline \multicolumn{9}{|l|}{ Stelechomyia (?) } \\
\hline \multicolumn{9}{|l|}{ Stenochironomus } \\
\hline Tanytarsus & & $0.6(2.3) 0.1$ & & & $2.4(6.8) 0.2$ & $38(81.4) 4.9$ & & $2 .(6.3) 0.3$ \\
\hline \multicolumn{9}{|l|}{ Tribelos } \\
\hline \multicolumn{9}{|l|}{ Zavreliella } \\
\hline \multicolumn{9}{|l|}{ Chironomini } \\
\hline \multicolumn{9}{|l|}{ Tanytarsini } \\
\hline \multicolumn{9}{|l|}{ Orthocladiinae } \\
\hline \multicolumn{9}{|l|}{ Corynoneura } \\
\hline Cricotopus & & & & $0.4(1.9) 0.3$ & & & & \\
\hline \multicolumn{9}{|l|}{ Lopescladius } \\
\hline \multicolumn{9}{|l|}{ Nanocladius } \\
\hline Total & $3(7.6)$ & $35.9(77)$ & $3.2(6.2)$ & $5.2(8)$ & $132.8(190)$ & $289.8(458.6)$ & $44.2(40.2)$ & $82.9(96.1)$ \\
\hline
\end{tabular}


TABLE 2 (continued)

\begin{tabular}{|c|c|c|c|c|c|c|}
\hline & Pla & Plb & Ppa & Ppb & Ima & Imb \\
\hline & $\chi(s) \mathrm{d}$ & $\chi(s) \mathrm{d}$ & $\chi(s) \mathrm{d}$ & $\chi(\mathrm{s}) \mathrm{d}$ & $\chi(s) \mathrm{d}$ & $\chi(\mathrm{s}) \mathrm{d}$ \\
\hline \multicolumn{7}{|l|}{ Tanypodinae } \\
\hline Ablabesmyia & & $0.9(2.8) 0.1$ & & $1.2(3.2) 0.2$ & & $1.8(3.8) 0.2$ \\
\hline Clinotanypus & & $0.3(1.7) 0.1$ & & & & \\
\hline Coelotanypus & $0.6(2.4) 0.1$ & $30.8(53.4) 3.7$ & & $0.4(1.9) 0.1$ & & \\
\hline Djalmabatista & & $0.3(1.7) 0.1$ & & & $2.7(4.7) 0.5$ & $21.7(27.6) 9$ \\
\hline Labrundinia & & $0.3(1.7) 0.1$ & & & $1.3(3.6) 0.1$ & \\
\hline Procladius & $0.6(2.4) 0.1$ & $12.9(27.8) 1$ & & $1.6(6.1) 0.2$ & $4.1(5.1) 1.2$ & $1.8(3.8) 0.1$ \\
\hline \multicolumn{7}{|l|}{ Chironominae } \\
\hline Aedokritus & & $4.8(20.6) 0.1$ & & & & \\
\hline Axarus & $4.8(16.9) 1.8$ & & & & $4.1(7.6) 0.8$ & $5.4(11.1) 1$ \\
\hline Beardius & & $0.3(1.7) 0.1$ & & & $1.3(3.6) 0.1$ & $3.0(7.6) 0.4$ \\
\hline Chironomиs & $12.6(21) \mathbf{1 2 . 2}$ & 103.5(304.6)10.6 & 7.2(11.7)25.1 & $2(4.9) 0.6$ & $2.7(7.3) 0.2$ & $0.6(2.4) 0.1$ \\
\hline Cladopelma & & $4.5(8.8) 0.2$ & & & & \\
\hline Cryptochironomus & & $6(10.9) 0.4$ & & & $4.1(5.1) 1.2$ & $22.9(31.9) \mathbf{1 1 . 7}$ \\
\hline \multicolumn{7}{|l|}{ Dicrotendipes } \\
\hline Fissimentum sp2 & & & & & $5.5(14.6) 0.5$ & $5.4(7.8) 1.5$ \\
\hline F. desiccatum & $1.2(3.3) 0.4$ & $0.3(1.7) 0.1$ & & & $11(25.2) 2.2$ & $4.8(10.5) 0.9$ \\
\hline Goeldichironomиs & $3.6(12.1) 1.4$ & $51(76.2) 6.2$ & $2.4(8.3) 1.6$ & $1.6(7.8) 0.1$ & & $1.2(4.8) 0.1$ \\
\hline Harnischia & & $0.3(1.7) 0.1$ & & & $4.1(7.6) 0.8$ & \\
\hline Nimbocera & & $4.2(18.8) 0.1$ & & $1.6(7.8) 0.1$ & & \\
\hline Parachironomus & & & & & $2.7(7.3) 0.2$ & \\
\hline Polypedilum & $8.4(18.9) 6.6$ & $233(518.8) 36.8$ & $1.6(3.7) 2.2$ & $43(202.8) \mathbf{1 0 . 3}$ & $17.9(20.4) 7.2$ & $12.6(16.8) 4.7$ \\
\hline Rheotanytarsus & & & & & $11(29.2) 1.1$ & $4.2(10.5) 0.6$ \\
\hline Saetheria (?) & & $0.3(1.7) 0.1$ & & & & \\
\hline Stelechomyia (?) & & & & & $2.7(7.3) 0.2$ & \\
\hline Stenochironomus & & & & & $1.3(3.6) 0.1$ & $0.6(2.4) 0.1$ \\
\hline Tanytarsus & & $0.9(2.8) 0.1$ & $0.8(2.7) 0.5$ & $0.4(1.9) 0.1$ & $12.4(22.8) 2.5$ & $22.3(33.9) 7.3$ \\
\hline Tribelos & & & & & & $10.2(24.8) 1.9$ \\
\hline Zavreliella & & $0.6(3.4) 0.1$ & & & & $0.6(2.4) 0.1$ \\
\hline Chironomini & & & & & & $0.6(2.4) 0.1$ \\
\hline Tanytarsini & & & & & & $0.6(2.4) 0.1$ \\
\hline \multicolumn{7}{|l|}{ Orthocladiinae } \\
\hline Corynoneura & & & & & $1.3(3.6) 0.1$ & $1.2(3.3) 0.1$ \\
\hline \multicolumn{7}{|l|}{ Cricotopus } \\
\hline Lopescladius & & & & & 49.6(89.3)15.1 & $10.8(26.3) 1.5$ \\
\hline Nanocladius & & & & & & $1.8(7.2) 0.1$ \\
\hline Total & $32(40.9)$ & $455.6(741.1)$ & $12(13.7)$ & $51.9(220)$ & $140.7(166.5)$ & $134.6(102.1)$ \\
\hline
\end{tabular}


The first two axes of PCA showed limnological differences between high water and low water periods and differences in the sediment composition between lagoons and rivers. The variables that contributed to the formation of the first principal component (PC1) separated Patos lagoon from the majority of the sampling sites in the Baía and Ivinheima Rivers, mainly with respect to sediment texture.

The bottom of this lagoon was composed basically of mud and organic matter, but some parts of the rivers had important sites with very coarse sand, coarse sand, medium sand and granules. The second principal component (PC2) separated the high water period with higher water levels, higher water temperatures, higher conductivity and greater depth from the low water period characterized by higher values of dissolved oxygen in most sampling sites (Fig. 3).

The results obtained by Detrended Correspondence Analysis (DCA), and based on sites/ months and chironomid densities, are shown in Fig. 4. The first and second axes of the DCA present eigenvalues 0.55 and 0.34 , respectively. The distribution of sites/months scores of the first two axis of DCA separated the chironomid fauna of the Ivinheima River from the Guaraná and Patos lagoons and the Baía River. In this way, two main groups could be distinguished. Group I was formed by the genera: Ablabesmyia, Coelotanypus, Procladius, Aedokritus, Axarus, Chironomus, Cladopelma, Goeldichironomus, Nimbocera, Parachironomus, Polypedilum, Saetheria and Tanytarsus, found in lagoons and Baía River. The genera Chironomus and Polypedilum were abundant mainly in Patos lagoon, while Procladius abounded in the Baía River. The other group (Group II) was composed of Djalmabatista, Cryptochironomus, Fissimentum desiccatum, Fissimentum sp2, Rheotanytarsus, Tribelos and Lopescladius in the Ivinheima River.

The results of the $m^{2}$ test showed a significant correlation between physical and chemical variables and chironomid larval densities $\left(m^{2}=0.9439\right.$ and $\mathrm{p}=0.0020)$ (Fig. 5).

\section{DISCUSSION}

According to Pinder (1986), the presence of aquatic macrophytes in aquatic ecosystems can result in an increase in the surface area for the colonisation of invertebrates; this has been confirmed by Poi de Neiff \& Carignan (1997) and Souza-Franco \& Takeda (2000).

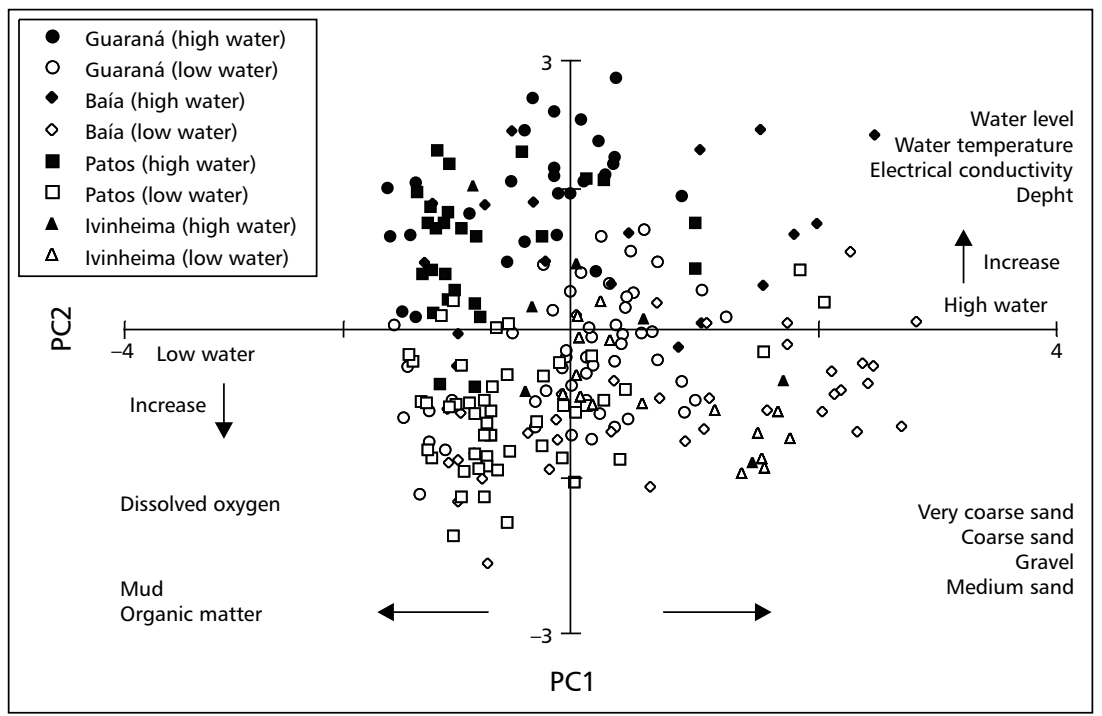

Fig. 3 - First two axes of the Principal Component Analysis (PCA) ordination diagram. 


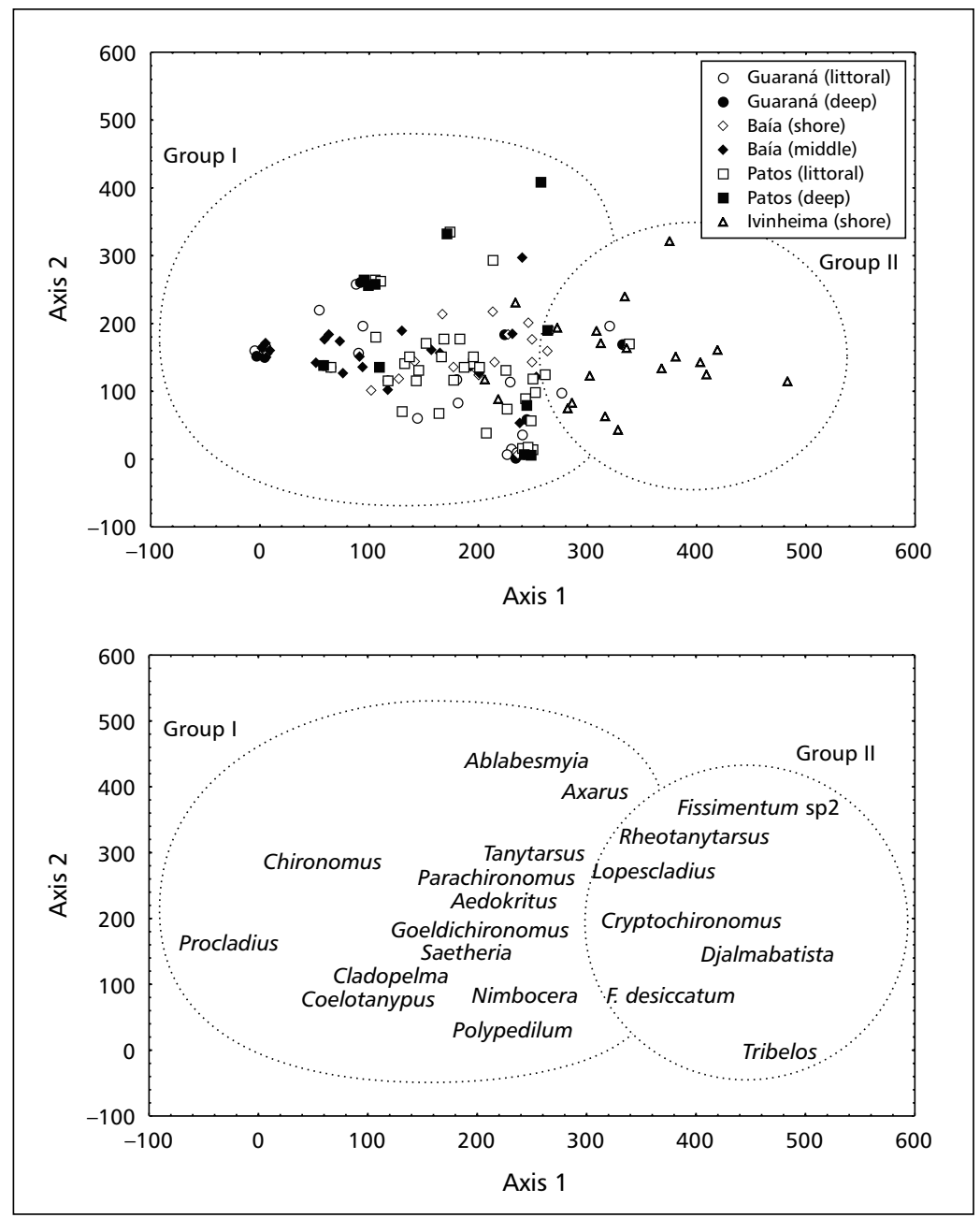

Fig. 4 - First two axes of the Detrended Correspondence Analysis (DCA) ordination diagram. (a) ordination of the enviroment/ month scores, (b) ordination of the chironomid genera.

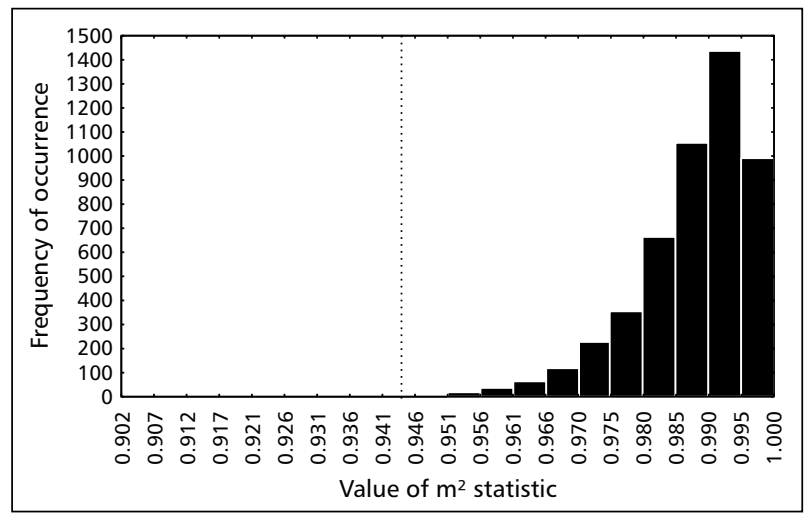

Fig. 5 - Distribution of $m^{2}$ statistic based on 4,999 randomized matrices derived from PCA and DCA scores. 
Aquatic macrophytes can be used as a substrate for the deposition of eggs (mainly of insects), as a refuge for various groups and can also contribute, with the formation of detritus, to create new microhabitats. Extensive stands of E. azurea in the Guaraná lagoon littoral region increase the available area of colonisation for invertebrates in these aquatic macrophytes. This fact, along with the low dissolved oxygen concentration in the bottom, were probably responsible for the lower benthic chironomid larvae mean density in Guaraná lagoon compared to Patos lagoon.

The detritivorous feeding habits of Polypedilum (Culp et al., 1983), along with the shallowness of the littoral region (particularly during low water period) probably causes the dominance of this genus in the muddy bottoms of the lagoons.

During high water periods, the decrease of the mean densities of chironomids can be related to the decrease in dissolved oxygen concentration and the increase in current velocity. During the inundation period, the increase in depth together with greater current speed, can lead to erosion on the bottom composed of sand or mud, thus decreasing organism densities with the erosion.

The oscillation in water level in the Ivinheima River had less influence on the chironomid assemblage in the Patos lagoon and this was probably due to the longer connecting channel, the greater extension of the lagoon and the less intense inundation pulse. The genus Chironomus dominated here during high water period.

The presence of pigment and abdominal tube on Chironomus larvae are characteristics important to balance the absence of the oxygen. That can explain its dominance during high water period, mainly in Patos lagoon profundal region due to the lower oxygen concentration and the greater percentage of organic matter. The high abundance of Chironomus in the profundal regions was also observed by Takeda et al. (1997) in Pousada das Garças and Fechada lagoons of in the same floodplain.

Although Goeldichironomus is more common in lentic habitats (Epler, 1992), it was dominant on the Baía River margins. Reiss (1977) and Ferrington \& Pehofer (1996), working with $G$. pictus and Goeldichironomus sp. respectively, showed a relationship between the variable depth and the presence/abundance of this genus. Its dominance in the Baía River margins can thus be due to the fact that this river presents hydrodynamics characteristics which are more similar to those of lagoons, while the sampling sites are also rather shallow.

The Procladius dominance in the Baía River central region, and this during both hydrological cycles, was probably related to the fact that the predominant sediment in this environment consisted mainly of fine sand with a layer of mud. The presence of mud probably influenced the dominance of this genus. This was also seen by Barton (1986) and Higuti et al. (1993) in muddy substrate and by Strixino \& Trivinho-Strixino (1991) in sandy-muddy sediment. This genus was also dominant on the Baía River margin and Guaraná lagoon littoral region during high water period. In a study undertaken by Hare (1995), Procladius was found mainly in the marginal region. This indicates that the larvae of this species can tolerate a wide variety of factors and are able in inhabit both marginal and central regions.

Due to different fluvial action on the Ivinheima River margins, the right margin suffers most erosive action, because of the greater current, and this is shown mainly by the pebble substrate, sand and the little accumulation of organic matter in the sediment. This seems to determine the dominance of Lopescladius and the presence of Rheotanytarsus. Both genera are typical of lotic environments (Epler, 1992; Pinder \& Reiss, 1983). Rheotanytarsus inhabits heterogeneous substrates, with little organic matter (Rae, 1985) and prefers rapid flux areas due to is filtering habits (Barmuta, 1990).

The Ivinheima River left margin, on the other hand, has a greater depositional activity and here, Cryptochironomus was found to be dominant on the sandy bottom. This genus is known to inhabit areas composed of fine sand (Grzybkowska \& Witczak, 1990; Higuti et al., 1993). It is thus probable that dominance of this genus was indeed due to the lotic habitat (depositional area) and sandy substrate in this area, a habitat also examined by Sanseverino \& Nessimian (2001).

In the Baía River study site, the current is slow and the sediment is composed of sand and mud laid over pebbles and granules; the environment is thus similar to a lentic one. This could explain the similarity between the composition of the chironomid fauna of the lagoons and of this river. 


\section{CONCLUSIONS}

Although the identification of the chironomid fauna could only be done down to the generic level, and this due to the fact that the taxonomy of Brazilian chironomid larvae is ill known (TrivinhoStrixino \& Strixino, 1995), the present paper has shown the importance of hydrodynamics and of the flood pulse on the spatial and temporal variation in density and dominance of chironomid larvae. The flood pulse fluctuation determines the temporal variation of limnological characteristics, such as dissolved oxygen, whereas the hydrodynamics have a great spatial effect on sediment composition, organic matter and presence or absence of aquatic macrophytes.

Acknowledgments - We would like to thank Dr. Luiz Felipe Machado Velho and Dr. Fábio Amodeo Lansac-Tôha for help with the sampling and valuable suggestions; Dr. Susana Trivinho-Strixino for help in the identification of the chironomid larvae and valuable comments; Dr. Koen Martens for reading the manuscript and for his valuable suggestions and comments; Dr. Luis Maurício Bini for statistical support; designer Jaime Luiz Lopes Pereira for the map; Physical and Chemical Laboratory of Nupélia for abiotic parameters; PADCT/CIAMB-CNPq and Nupélia for grants and support.

\section{REFERENCES}

AMOROS, C., 1991, Changes in side-arm connectivity and implications for river system management. Rivers, 2: 105-112.

BARMUTA, L. A., 1990, Interaction between the effects of substratum, velocity and location on stream benthos: an experiment. Aust. J. Mar. Freshwat. Res., 41: 557-573.

BARTON, D. R., 1986, Invertebrates of the mackenzie system, pp. 473-492. In: B. R. Davies \& K. F. Walker (eds.), The ecology of river systems. W. Junk Publishers, Dordrecht.

BONETTO, A. A., 1993, Structure and functioning of large river floodplains of neotropical America: the ParanaParaguay system, pp. 123-138. In: B. Gopal, A. Hillbricht-Llkowska \& R. G. Wetzel (eds.), Wetlands and ecotones. National Institute of Ecology, New Delhi.

COFFMAN, W. P. \& FERRINGTON Jr., L. C., 1988, Chironomidae, pp. 551-652. In: R. W. Merritt \& K. W. Cummins (eds.), An Introduction to the aquatic insects of North America. Kendall, Hunt Publishing, Dubuque, Iowa.

CUlP, J. M., WALDE, S. J. \& DAVIES, R. W., 1983, Relative importance of substrate particle size and detritus to stream benthic macroinvertebrate microdistribution. Can. J. Fish. Aquat. Sci., 40: 1568-1574.
DNAEE, Departamento Nacional de Águas e Energia Elétrica. Dados hidrométricos e pluviométricos diários. Estação de Porto São José, PR. Boletim Informativo.

DOKULIL, M. T. \& SCHIEL, G., 2000, The sediment-water interface as an ecotone: an example from an oxbow lake of the River Danube. Verh. Internat. Verein. Limnol., 27: 402-405.

EPLER, J. H., 1992, Identification manual for the larval Chironomidae (Diptera) of Florida. Florida Department of Environmental Regulation, Orlando, Florida, 301p.

FERRINGTON Jr., L. C. \& Pehofer, H. E., 1996, Instar distribution and biomass of Chironomidae larvae in Lago El Junco, Isla San Cristobal, the Galápagos. Hydrobiologia, 318: 123-133.

GOLTERMAN, H. L., CLYMO, R. S. \& OHNSTAD, M. A. M., 1978, Methods for physical and chemical analysis of fresh waters. Blackwell Scientific, Oxford.

GRZYBKOWSKA, M. \& WITCZAK, J., 1990, Distribution and production of Chironomidae (Diptera) in the lower course of the Grabia River (Central Poland). Freshwat. Biol., 24: 519-531.

HARE, L., 1995, Sediment colonization by littoral and profundal insects. J. North Am. Benthol. Soc., 14(2): 315-323.

HIGUTI, J., TAKEDA, A. M. \& PAGGI, A. C., 1993, Distribuição espacial das larvas de Chironomidae (Insecta, Diptera) do rio Baía (MS-Brasil). Rev. UNIMAR, 15(suplemento): 65-81.

JACKSON, D. A., 1995, Protest: a procrustean randomization test of community environment concordance. Ecoscience, 2: 297-303.

JUNK, W. J., BAYLEY, P. B. \& SPARKS, R. E., 1989, The flood pulse concept in river-floodplain systems. Can. Spec. Publ. Fish. Aquat. Sci., 106: 110-127.

KOWNACKI, A., 1971, Taxocens of Chironomidae in streams of the Polish High Trata Mts. Acta Hydrobiol., 13(4): 439-464

MENZIE, C. A., 1981, Production ecology of Cricotopus sylvestris (Fabricius) (Diptera: Chironomidae) in a shallow estuarine cove. Limnol. Oceanogr., 26(3): 467-481.

PINDER, L. C. V., 1986, Biology of freshwater Chironomidae. Ann. Rev. Ent., 31: 1-23.

PINDER, L. C. V. \& REISS, F., 1983, The larvae of Chironominae (Diptera: Chironomidae) of the Holartic region - keys and diagnoses, pp. 293-435. In: T. Wiederholm (ed.), Chironomidae of the Holartic Region: keys and diagnoses. Part 1 - Larvae. Borgströms Tryckeri AB, Montala, Entomologica Scandinavica, supplement 19.

POI DE NEIFF, A. \& CARIGNAN, R., 1997, Macroinvertebrates on Eichhornia crassipes roots in two lakes nof the Paraná River floodplain. Hydrobiologia, 345: 185-196.

RAE, J. G., 1985, A multivariate study of resource partitioning in soft bottom lotic Chironomidae. Hydrobiologia, 126: 275-285. 
REISS, F., 1977, The benthic zoocoenoses of Central Amazon varzea lakes e their adaptations to the annual water level fluctuations. Geogr. Ecol. Trop., 1(2): 65-75.

SANSEVERINO, A. M. \& NESSIMIAN, J. L., 2001, Habitats de larvas de Chironomidae (Insecta, Diptera) em riachos de Mata Atlântica no Estado do Rio de Janeiro. Acta Limnol. Bras., 13(1): 29-38.

SOUZA-FRANCO, G. M. \& TAKEDA, A. M., 2000, Invertebrates associated with Paspalum repens (Poaceae) at the mouth of Caracu stream (1991-1992), affluent of the Paraná River, Porto Rico-PR-Brazil. Brazil. Arch. Biol. Technol., 43(3): 317-325.

STRIXINO, G. \& TRIVINHO-STRIXINO, S., 1991, Chironomidae (Diptera) associados a sedimentos de reservatórios: significado dos diferentes povoamentos. In: Anais do Seminário Regional de Ecologia, 6: 151-168.

SUGUIO, K., 1973, Introdução à sedimentologia. Edgard Blücher, São Paulo, 317p.

TAKEDA, A. M., SHIMIZU, G. Y. \& HIGUTI, J., 1990, Zoobentos de uma lagoa marginal (lagoa Fechada, rio Baía, Alto Paraná, PR). Cienc. Cult., 42(11): 1003-1007.

TAKEDA, A. M., SAMPAIO, A. A., YAMAMOTO, M. M. \& HIGUTI, J., 1991a, Zoobentos do rio Baía, MS (alto rio Paraná, Brasil). Rev. UNIMAR, 13(2): 339-352.
TAKEDA, A. M., SHIMIZU, G. Y., SHULZ, G. M. \& SILVA, A. C. M., 1991b, Zoobentos de quatro lagoas de várzea do alto rio Paraná (MS-Brasil). Influência do regime hidrológico sobre a comunidade. Rev. UNIMAR, 13(2): 365-387.

TAKEDA, A. M., SHIMIZU, G. Y. \& HIGUTI, J., 1997, Variações espaço-temporais da comunidade zoobêntica, pp. 157-177. In: A. E. A. M. Vazzoler, A. A. Agostinho \& N. S. Hahn (eds.), A planície de inundação do alto rio Paraná. EDUEM, Maringá.

THOMAZ, S. M., ROBERTO, M. C. \& BINI, L. M., 1997, Caracterização limnológica dos ambientes aquáticos e influência dos níveis fluviométricos, pp. 73-102. In: A. E. A. M. Vazzoler, A. A. Agostinho \& N. S. Hahn (eds.), A planície de inundação do alto rio Paraná. EDUEM, Maringá.

TRIVINHO-STRIXINO, S. \& STRIXINO, G., 1995, Larvas de Chironomidae (Diptera) do Estado de São Paulo. Guia de identificação e diagnose dos gêneros. Universidade Federal de São Carlos, Programa de PósGraduação em Ecologia e Recursos Naturais, São Carlos, $229 \mathrm{p}$.

WENTWORTH, C. K., 1922, A scale of grade and class terms for clastic sediments. J. Geol., 30: 377-392. 